hep-ph/0305212

CERN-TH/2003-107

UMN-TH-2201/03

FTPI-MINN-03/12

\title{
Phenomenological Constraints on Patterns of Supersymmetry Breaking
}

\author{
John Ellis $^{1}$, Keith A. Olive ${ }^{2}$, Yudi Santoso ${ }^{2}$ and Vassilis C. Spanos ${ }^{2}$ \\ ${ }^{1}$ TH Division, CERN, Geneva, Switzerland \\ ${ }^{2}$ William I. Fine Theoretical Physics Institute, \\ University of Minnesota, Minneapolis, MN 55455, USA
}

\begin{abstract}
Specific models of supersymmetry breaking predict relations between the trilinear and bilinear soft supersymmetry breaking parameters $A_{0}$ and $B_{0}$ at the input scale. In such models, the value of $\tan \beta$ can be calculated as a function of the scalar masses $m_{0}$ and the gaugino masses $m_{1 / 2}$, which we assume to be universal. The experimental constraints on sparticle and Higgs masses, $b \rightarrow s \gamma$ decay and the cold dark matter density $\Omega_{C D M} h^{2}$ can then be used to constrain tan $\beta$ in such specific models of supersymmetry breaking. In the simplest Polonyi model with $A_{0}=(3-\sqrt{3}) m_{0}=B_{0}+m_{0}$, we find $11 \lesssim \tan \beta \lesssim 20(\tan \beta \simeq 4.15)$ for $\mu>0(\mu<0)$. We also discuss other models with $A_{0}=B_{0}+m_{0}$, finding that only the range $-1.9 \lesssim A_{0} / m_{0} \lesssim 2.5$ is allowed for $\mu>0$, and the range $1.25 \lesssim A_{0} / m_{0} \lesssim 4.8$ for $\mu<0$. In these models, we find no solutions in the rapid-annihilation 'funnels' or in the 'focus-point' region. We also discuss the allowed range of $\tan \beta$ in the no-scale model with $A_{0}=B_{0}=0$. In all these models, most of the allowed regions are in the $\chi-\tilde{\tau}_{1}$ coannihilation 'tail'.
\end{abstract}

CERN-TH/2003-107

May 2003 


\section{Introduction}

One of the most important and least understood problems in the construction of supersymmetric models is the mechanism of supersymmetry breaking [1]. Direct exploration of this may be far beyond our experimental reach for some considerable time, so we may have to rely on indirect information provided by measurements of the different soft supersymmetrybreaking parameters. Even here, so far we have no determinations, only limits obtained from accelerator experiments, cosmology and theoretical considerations. It is commonly assumed that the soft supersymmetry-breaking scalar masses $m_{0}$ have universal values at some GUT input scale, as do the gaugino masses $m_{1 / 2}$ and the trilinear soft supersymmetry-breaking parameters $A_{0}$, which is referred to as the constrained MSSM (CMSSM). One then frequently analyzes the impacts of the different phenomenological limits on the allowed values of $m_{1 / 2}$ and $m_{0}$ as functions of $\tan \beta$, the ratio of Higgs vacuum expectation values, assuming some default value of $A_{0}$ and determining the Higgs mixing parameter $\mu$ and the pseudoscalar Higgs mass $m_{A}$ by using the electroweak vacuum consistency conditions (see [2] - [11] for recent studies of this type). The tree-level value of $m_{A}$ may be related to the bilinear soft supersymmetry-breaking parameter $B$, via $m_{A}^{2}=-2 B \mu / \sin 2 \beta$.

Specific models of supersymmetry breaking predict relations between these different soft supersymmetry-breaking parameters. For example, certain 'no-scale' models [12] may predict $m_{0}=0$ at the Planck scale, and we have analyzed the extent to which this assumption is compatible with the phenomenological constraints, taking account of the possible running of $m_{0}$ between the Planck scale and the GUT scale [13]. Here we analyze a different question, namely the consistency of some proposed relations between $m_{0}, A_{0}$ and $B_{0}$ which take the characteristic form

$$
A_{0}=\hat{A} m_{0}, \quad B_{0}=\hat{B} m_{0}
$$

A generic minimal supergravity model [14] prediction is that $\hat{B}=\hat{A}-1[15]$, and the simplest Polonyi model [16] predicts that $|\hat{A}|=3-\sqrt{3}[17]$.

The first of the two relations (1) may be used to replace an ad hoc assumption on the input value of $A_{0}$. The second imposes an important consistency condition on the value of $m_{A}$, which was otherwise treated as a dependent quantity that was not constrained $a$ priori. For any given value of $m_{1 / 2}$ and $m_{0}$, this constraint is satisfied for only one specific value of $\tan \beta$. Therefore, the results of imposing the two constraints (1) may conveniently be displayed in a single $\left(m_{1 / 2}, m_{0}\right)$ plane across which $\tan \beta$ varies in a determined manner. The phenomenological constraints on $m_{1 / 2}$ and $m_{0}$ can then be used to provide both upper and lower limits on the allowed values of $\tan \beta$. 
In this paper, we analyze these constraints on $\tan \beta$ as functions of $\hat{A}$ in the generic scenario (1), including the Polonyi case $\hat{A}=3-\sqrt{3}$ and other models with $\hat{A}=\hat{B}+1$. In the Polonyi case, we find that $11 \lesssim \tan \beta \lesssim 20$ for $\mu>0$, with only a small area in the $m_{1 / 2}-m_{0}$ plane with $\tan \beta \simeq 4.15$ surviving for $\mu<0$. In general, we find consistent solutions for $-1.9 \lesssim \hat{A} \lesssim 2.25$ for $\mu>0$ and $1.25 \lesssim \hat{A} \lesssim 4.8$ for $\mu<0$. We also explore the range of $\tan \beta$ that is allowed in a no-scale scenario with $A_{0}=B_{0}=0$ at the GUT scale. It should, however, be recalled that the no-scale boundary conditions [12] were originally proposed to hold at the supergravity scale, which might be significantly above the GUT scale. In this case, renormalization-group running between these scales would generate $\hat{A}$ and $\hat{B} \neq 0$ at the GUT scale.

\section{Models of Supersymmetry Breaking}

In this Section, we review briefly models that yield the characteristic patterns of supersymmetry breaking whose phenomenology we study later in the paper. We assume an $N=1$ supergravity framework, interpreted as a low-energy effective field theory. This may be characterized by a Kähler function $K$ that describes the kinetic terms for the chiral supermultiplets $\Phi \equiv(\zeta, \phi)$, where the $\zeta$ represent hidden-sector fields and the $\phi^{i}$ observable-sector fields, a holomorphic function $f(\Phi)$ that yields kinetic terms for the gauge supermultiplets $A_{a}$ as well as gauge couplings, and a holomorphic superpotential $W(\Phi)$. We assume the form of the gauge kinetic function $f$ to be such that the gaugino masses $m_{1 / 2}$ are universal at the GUT input scale, as are the gauge couplings.

So-called minimal supergravity theories have $K=\Sigma_{i}\left|\Phi^{i}\right|^{2}$, whereas no-scale models have non-trivial Kähler functions such as $K=-3 \ln \left(\zeta+\zeta^{\dagger}-\Sigma_{j}\left|\phi^{j}\right|^{2}\right)$. The scalar potential (neglecting any gauge contributions) is in general [14]

$$
V\left(\phi, \phi^{*}\right)=e^{K}\left[K^{i}\left(K^{-1}\right)_{i}^{j} K_{j}-3\right]
$$

where we are working in Planck units. For minimal supergravity, we have $K^{i}=\phi^{i^{*}}+W^{i} / W$, $K_{i}=\phi_{i}+W_{i}^{*} / W^{*}$, and $\left(K^{-1}\right)_{i}^{j}=\delta_{i}^{j}$, and the resulting scalar potential is

$$
V\left(\phi, \phi^{*}\right)=e^{\phi_{i} \phi^{i *}}\left[\left|W^{i}+\phi^{i^{*}} W\right|^{2}-3|W|^{2}\right] .
$$

In this minimal case, the soft supersymmetry-breaking scalar masses $m_{0}$ are universal at the input GUT scale, with [1]

$$
m_{0}^{2}=m_{3 / 2}^{2}+\Lambda
$$


where $m_{3 / 2}$ is the gravitino mass and $\Lambda$ is the tree-level cosmological constant. If we further assume that the superpotential $W(\Phi)$ may be separated into pieces $F$ and $g$ that are functions only of observable-sector fields $\phi^{i}$ and hidden-sector fields $\zeta$, respectively, so that the superpotential parameters of the observable-sector fields do not depend on the hidden-sector fields, then the trilinear terms $A_{0}$ and bilinear terms $B_{0}$ are also universal, and [1]

$$
B_{0}=A_{0}-m_{3 / 2}
$$

Finally, if we further assume that $\Lambda=0$, then $m_{0}=m_{3 / 2}$ and [1]

$$
\hat{B}=\hat{A}-1
$$

which is one of the principal options we study below.

One of the primary motivations for the CMSSM, and for scalar mass universality in particular, comes from the simplest model for local supersymmetry breaking [16], which involves just one additional chiral multiplet $\zeta$ in addition to the observable matter fields $\phi_{i}$. We consider, therefore, a superpotential which is separable in this so-called Polonyi field and the $\phi_{i}$, and of the simple form

$$
g(\zeta)=\nu(\zeta+\beta)
$$

with $|\beta|=2-\sqrt{3}$, ensuring that $\Lambda=0$. The scalar potential in this model takes the form [17]

$$
\begin{aligned}
V= & e^{\left(|\zeta|^{2}+|\phi|^{2}\right)}\left[\left|\frac{\partial g}{\partial \zeta}+\zeta^{*}(g(\zeta)+F(\phi))\right|^{2}\right. \\
& \left.+\left|\frac{\partial F}{\partial \phi}+\phi^{*}(g(\zeta)+F(\phi))\right|^{2}-3|g(\zeta)+F(\phi)|^{2}\right]
\end{aligned}
$$

We next expand the expression (8) and drop terms that are suppressed by inverse powers of the Planck scale, which can be done simply by dropping terms of mass dimension greater than four. In the positive case, after inserting the vev for $\zeta,\langle\zeta\rangle=\sqrt{3}-1$, we have [17]:

$$
\begin{aligned}
V= & e^{(4-2 \sqrt{3})}\left[|\nu+(\sqrt{3}-1)(\nu+F(\phi))|^{2}\right. \\
& \left.+\left|\frac{\partial F}{\partial \phi}+\phi^{*}(\nu+F(\phi))\right|^{2}-3|\nu+F(\phi)|^{2}\right] \\
= & e^{(4-2 \sqrt{3})}\left|\frac{\partial F}{\partial \phi}\right|^{2} \\
& \left.+m_{3 / 2} e^{(2-\sqrt{3})}\left(\phi \frac{\partial F}{\partial \phi}-\sqrt{3} F+\text { h.c. }\right)\right)+m_{3 / 2}^{2} \phi \phi^{*},
\end{aligned}
$$

which deserves some discussion. 
First, up to an overall rescaling of the superpotential, $F \rightarrow e^{\sqrt{3}-2} F$, the first term is the ordinary $F$-term part of the scalar potential of global supersymmetry. The next term, which is proportional to $m_{3 / 2}$, provides universal trilinear soft supersymmetry-breaking terms $A=(3-\sqrt{3}) m_{3 / 2}$ and bilinear soft supersymmetry-breaking terms $B=(2-\sqrt{3}) m_{3 / 2}$, i.e., a special case of the general relation (5) above between $B$ and $A$. Finally, the last term represents a universal scalar mass of the type advocated in the CMSSM, with $m_{0}^{2}=m_{3 / 2}^{2}$, since the cosmological constant $\Lambda$ vanishes in this model, by construction.

As we have seen above, the generation of such soft terms is a rather generic property of low-energy supergravity models [15] and many of these conclusions persist when one generalizes the Polonyi potential. For example, if we choose $g(\zeta)$ so that ${ }^{1}\langle g\rangle=\nu,\langle\partial g / \partial \zeta\rangle=$ $a^{*} \nu$, and $\langle\zeta\rangle=b$, the condition that $\Lambda=0$ at $\zeta=b$ implies $|a+b|^{2}=3$. Substituting these expectation values in (8), we find [15] that $A=b^{*}(a+b) \nu$ and once again $B=A-\nu$, but now with $A$ free. The constant $\nu$ determines the gravitino mass, and hence $m_{0}$, through: $m_{0}=m_{3 / 2}=e^{\frac{1}{2} b b^{*}} \nu$.

Another broad option for supersymmetry breaking is that provided by no-scale models [12], of which the simplest example is

$$
K=-3 \ln \left(\zeta+\zeta^{\dagger}-\Sigma_{i}\left|\phi^{i}\right|^{2}\right)
$$

No-scale models have the universal values

$$
m_{0}^{2}=0, A_{0}=0, B_{0}=0
$$

at the input supergravity scale. The possibility that $m_{0}=0$ at the GUT scale has recently been studied $[13,18]$, and shown to be excluded by the phenomenological constraints. However, it was recalled that the input supergravity scale could be somewhat higher than the GUT scale, in which case one might find $m_{0} \neq 0$ already at the GUT scale. Clearly the same could also be true for $A_{0}$ and $B_{0}$. However, the deviations from (11) are modeldependent, and we think it important to be aware of the phenomenological fate of the clear-cut $A_{0}=B_{0}=0$ option for supersymmetry breaking.

\section{Electroweak Vacuum Conditions}

Before discussing the phenomenological constraints on this model, we first show more precisely how the relation between $A$ and $B$ can be used to determine $\tan \beta$ when the radiative electroweak symmetry breaking conditions are applied.

\footnotetext{
${ }^{1}$ One could also consider models in which several fields $\zeta_{i}$ contribute to supersymmetry breaking.
} 
In general, we start with the following set of input parameters defined at the GUT scale: $m_{1 / 2}, m_{0}, A_{0}, B_{0}$ and the Higgs mixing parameter $\mu_{0}$. By running the full renormalizationgroup equations (RGEs) down to the weak scale and minimizing the Higgs potential, one can solve for the Higgs vevs and masses or, equivalently, $M_{Z}, \tan \beta$, and $m_{A}$. At the tree level, these solutions take the simple form:

$$
\begin{aligned}
M_{Z}^{2} & =\frac{2\left(m_{1}^{2}+\mu^{2}-\left(m_{2}^{2}+\mu^{2}\right) \tan ^{2} \beta\right)}{\left(\tan ^{2} \beta-1\right)} \\
\sin 2 \beta & =-2 B \mu /\left(m_{1}^{2}+m_{2}^{2}+2 \mu^{2}\right) \\
m_{A}^{2} & =m_{1}^{2}+m_{2}^{2}+2 \mu^{2}
\end{aligned}
$$

where $m_{1}$ and $m_{2}$ are the soft supersymmetry-breaking masses for the two Higgs doublets at the electroweak scale. However, since $M_{Z}$ is known, and because the full one-loop set of tadpole equations does not admit an analytical solution for $\tan \beta$, it is customary to use $M_{Z}$ and $\tan \beta$ as inputs and instead solve for $\mu$ and $B$ :

$$
\begin{aligned}
\mu^{2} & =\frac{m_{1}^{2}-m_{2}^{2} \tan ^{2} \beta+\frac{1}{2} m_{z}^{2}\left(1-\tan ^{2} \beta\right)+\Delta_{\mu}^{(1)}}{\tan ^{2} \beta-1+\Delta_{\mu}^{(2)}} \\
B \mu & =-\frac{1}{2}\left(m_{1}^{2}+m_{2}^{2}+2 \mu^{2}\right) \sin 2 \beta+\Delta_{B}
\end{aligned}
$$

where $\Delta_{B}$ and $\Delta_{\mu}^{(1,2)}$ are loop corrections [19, 20, 21], and here $m_{1,2} \equiv m_{1,2}\left(m_{Z}\right)$. Since $\Delta_{\mu}$ depends on $\tan \beta$ and $\Delta_{B}$ depends on both $\mu$ and $\tan \beta$ in a nonlinear way, it is not possible to write down an analytical solution for $\tan \beta$. The above set of inputs and outputs defines the CMSSM.

In the types of models discussed in the previous section, we have specific GUT-scale boundary conditions on $B_{0}$, namely $B_{0}=A_{0}-m_{0}$ in minimal supergravity models or $B_{0}=A_{0}=0$ in no-scale models. Therefore, we cannot treat the value of $B\left(M_{Z}\right)$ as a free parameter, and instead must solve numerically for $\tan \beta$. Thus, a given value of $m_{1 / 2}, m_{0}$, $A_{0} / m_{0}$, and $\operatorname{sgn}(\mu)$ will correspond to a definite value for $\tan \beta$. When combined with the phenomenological constraints discussed below, we can determine for a particular model of supersymmetry breaking the allowed (and often quite restricted) values of $\tan \beta$.

\section{Phenomenological Constraints on $m_{1 / 2}$ and $m_{0}$}

We apply the standard LEP constraints on the supersymmetric parameter space, namely $m_{\chi^{ \pm}}>104 \mathrm{GeV}$ [22], $m_{\tilde{e}}>99 \mathrm{GeV}$ [23] and $m_{h}>114 \mathrm{GeV}$ [24]. The former two constrain $m_{1 / 2}$ and $m_{0}$ directly via the sparticle masses, and the latter indirectly via the sensitivity of 
radiative corrections to the Higgs mass to the sparticle masses, principally $m_{\tilde{t}, \tilde{b}}{ }^{2}$. We use the latest version of FeynHiggs [25] for the calculation of $m_{h}$. We require the branching ratio for $b \rightarrow s \gamma$ to be consistent with the experimental measurements [26]. We also indicate the regions of the $\left(m_{1 / 2}, m_{0}\right)$ plane that are favoured by the BNL measurement [27] of $g_{\mu}-2$ at the $2-\sigma$ level, corresponding to a deviation of $(33.9 \pm 11.2) \times 10^{-10}$ from the Standard Model calculation of [28] using $e^{+} e^{-}$data. We are however aware that this constraint is still under discussion and do not use it to constrain $\tan \beta$. All the $\mu>0$ planes would be consistent with $g_{\mu}-2$ at the $3-\sigma$ level, whereas $\mu<0$ is disfavoured even if one takes a relaxed view of the $g_{\mu}-2$ constraint.

Finally, we impose the following requirement on the relic density of neutralinos $\chi: 0.094 \leq$ $\Omega_{\chi} h^{2} \leq 0.129$, as suggested by the recent WMAP data [29], in agreement with earlier indications. We recall that several cosmologically-allowed domains of the $\left(m_{1 / 2}, m_{0}\right)$ planes for different values of $\tan \beta$ have been discussed previously in the general CMSSM framework [2] - [4], [6] - [11]. One is a 'bulk' region at low $m_{1 / 2}$ and $m_{0}$, which has been squeezed considerably by the WMAP constraint on $\Omega_{\chi} h^{2}$. A second region is the $\chi-\tilde{\tau}_{1}$ coannihilation 'tail' $[7,8]$, which stretches to larger $m_{1 / 2}$, close to the boundary of the acceptable region where $m_{\chi} \leq m_{\tilde{\tau}_{1}}$. In the wake of WMAP, this 'tail' is now much narrower - because of the smaller range of $\Omega_{\chi} h^{2}$ - and shorter - because of the more stringent upper limit on $\Omega_{\chi} h^{2}$ $[5,30]$. A third region is the 'funnel' due to rapid $\chi \chi \rightarrow H, A$ annihilation that occurs at larger $m_{0}$ and $m_{1 / 2}[4,9]$. Finally, the fourth domain is the 'focus-point' region at large $m_{0}$, close to the boundary where radiative breaking of electroweak symmetry is no longer possible [10, 11].

We see in the next Section that the 'funnel' and 'focus-point' regions are not present in the simple models of supersymmetry breaking introduced earlier, whilst the 'bulk' region is possible only for a very restricted range of $\tan \beta$. On the other hand, the coannihilation 'tail' generally remains permitted.

\section{Examples of $\left(m_{1 / 2}, m_{0}\right)$ Planes}

We display in Fig. 1 the contours of $\tan \beta$ (solid blue lines) in the $\left(m_{1 / 2}, m_{0}\right)$ planes for selected values of $\hat{A}, \hat{B}$ and the sign of $\mu$. Also shown are the contours where $m_{\chi^{ \pm}}>104 \mathrm{GeV}$ (near-vertical black dashed lines) and $m_{h}>114 \mathrm{GeV}$ (diagonal red dash-dotted lines). The excluded regions where $m_{\chi}>m_{\tilde{\tau}_{1}}$ have dark (red) shading, those excluded by $b \rightarrow s \gamma$ have medium (green) shading, and those where the relic density of neutralinos lies within the

\footnotetext{
${ }^{2}$ We assume as our default that $m_{t}=175 \mathrm{GeV}$.
} 
WMAP range $0.094 \leq \Omega_{\chi} h^{2} \leq 0.129$ have light (turquoise) shading. Finally, the regions favoured by $g_{\mu}-2$ at the $2-\sigma$ level are medium (pink) shaded.

As seen in panel (a) of Fig. 1 , when $\mu>0$ and $\hat{A}=-1.5$, close to its minimum possible value, the contours of $\tan \beta$ rise diagonally from low values of $\left(m_{1 / 2}, m_{0}\right)$ to higher values, with higher values of $\tan \beta$ having lower values of $m_{0}$ for a given value of $m_{1 / 2}$. The $m_{h}=114 \mathrm{GeV}$ contour rises in a similar way, and regions above and to the left of this contour have $m_{h}<114$ $\mathrm{GeV}$ and are excluded. Therefore, only a very limited range of $\tan \beta \sim 4$ is compatible with the $m_{h}$ and $\Omega_{C D M} h^{2}$ constraints. At lower values of $\hat{A}$, the slope of the Higgs contour softens and even less of the parameter space is allowed. Below $\hat{A} \simeq-1.9$, the entire $m_{1 / 2}-m_{0}$ plane is excluded. When $\hat{A}$ is increased to 0.75 , as seen in panel (b) of Fig. 1, both the $\tan \beta$ and $m_{h}$ contours rise more rapidly with $m_{1 / 2}$, and a larger range $9 \lesssim \tan \beta \lesssim 14$ is allowed ${ }^{3}$. In the simplest Polonyi model with $\hat{A}=3-\sqrt{3}$ shown in panel (c) of Fig. 1 , we see that the $\tan \beta$ contours have noticeable curvature. In this case, the Higgs constraint combined with the relic density requires $\tan \beta \gtrsim 11$, whilst the relic density also enforces $\tan \beta \lesssim 20^{4}$. Finally, in panel (d) of Fig. 1 , when $\hat{A}=2.0$, close to its maximal value for $\mu>0$, the $\tan \beta$ contours turn over towards smaller $m_{1 / 2}$, and only relatively large values $25 \lesssim \tan \beta \lesssim 35$ are allowed by the $b \rightarrow s \gamma$ and $\Omega_{C D M} h^{2}$ constraints, respectively.

In the case of $\mu<0$, negative values of $\hat{A}$ are not allowed, and only a tiny area in the $\left(m_{1 / 2}, m_{0}\right)$ plane near the end point of the coannihilation tail around $m_{1 / 2}=1000 \mathrm{GeV}$ is allowed in the positive Polonyi case $\hat{A}=3-\sqrt{3}$, as seen in panel (a) of Fig. 2. This is because the Higgs and $\Omega_{C D M} h^{2}$ constraints are barely compatible in this case, and allow only $\tan \beta \simeq 4.15$. At larger values of $\hat{A}$, the allowed region is extended, as exemplified in panel (b) of Fig. 2 for the case $\hat{A}=2.0$, where a small region around $\tan \beta \simeq 5.5-5.7$ is allowed. This panel shows that, approximately, the value of $\tan \beta$ depends only on the ratio $m_{1 / 2} / m_{0}[32]$.

There are several generic patterns in the results above that can be explained qualitatively, as follows. First, we notice that for any given value of $\left(m_{1 / 2}, m_{0}\right), \tan \beta$ increases as $\hat{A}$ increases. The reason for this can be found by looking at the second equation of (12), and setting $A_{0}=B_{0}+m_{0}$. For large $\tan \beta, \sin 2 \beta \sim 1 / \tan \beta$, so $B$ at the weak scale is inversely proportional to $\tan \beta$, at the tree level. In the $\mu>0$ case, this tree-level value of $B$ is negative, so its value grows as $\tan \beta$ increases. While loop corrections are generally negative for $\mu>0$, and RGE corrections to obtain $B\left(M_{X}\right)$ are positive, the monotonic growth of $B_{0}$

\footnotetext{
${ }^{3}$ Note that the contours for given values of $\tan \beta$ always intersect the axis $m_{0}=0$ at the same value of $m_{1 / 2}$.

${ }^{4}$ The other Polonyi case with $\hat{A}=-3+\sqrt{3}$ (not shown) is very similar to panel (a) for $\hat{A}=-1.5$, and has a very narrow allowed range of $\tan \beta \sim 4.5$.
} 

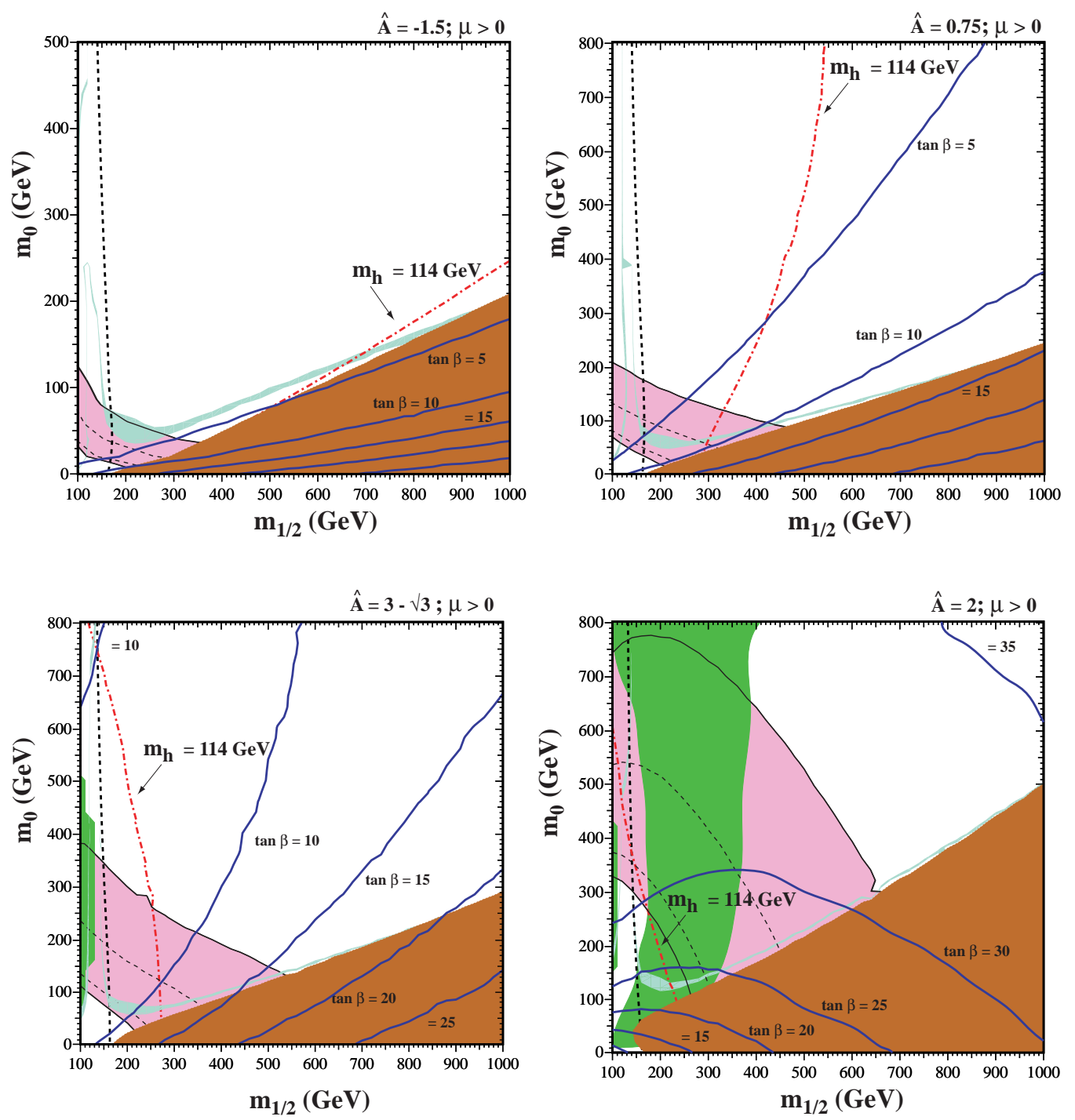

Figure 1: Examples of $\left(m_{1 / 2}, m_{0}\right)$ planes with contours of $\tan \beta$ superposed, for $\mu>0$ and (a) $\hat{A}=-1.5, \hat{B}=\hat{A}-1$, (b) $\hat{A}=0.75, \hat{B}=\hat{A}-1$, (c) the simplest Polonyi model with $\hat{A}=3-\sqrt{3}, \hat{B}=\hat{A}-1$ and (d) $\hat{A}=2.0, \hat{B}=\hat{A}-1$. In each panel, we show the regions excluded by the LEP lower limits on MSSM particles, those ruled out by $b \rightarrow s \gamma$ decay [26] (medium green shading), and those excluded because the LSP would be charged (dark red shading). The region favoured by the WMAP range $\Omega_{C D M} h^{2}=0.1126_{-0.0091}^{+0.0081}$ has light turquoise shading. The region suggested by $g_{\mu}-2$ is medium (pink) shaded. 
with $\tan \beta$ is preserved. Thus the resulting value of $B_{0}$, and hence also $A_{0}$, increases with $\tan \beta$. In the $\mu<0$ case, the tree-level value of $B$ is generally positive (the exception being when $m_{1}^{2}+m_{2}^{2}+2 \mu^{2}<0$ ), and so its value decreases as $\tan \beta$ increases. However, there are some terms in the loop correction $\Delta_{B}$ that are proportional to $\mu \tan \beta$ and flip the sign of $\Delta_{B}$ at a particular value of $\tan \beta$, so that the full one-loop $B\left(M_{W}\right)$ is then again an increasing function of $\tan \beta$, and likewise $A_{0}$.

Using similar arguments, we can further understand the different behaviours of the $\tan \beta$ contours when $\mu$ is positive or negative with fixed $\hat{A}$, for example in the last panels in Fig. 1 and Fig. 2 for $\hat{A}=2$. To this end, look at the second equation in (13), bearing in mind that $\sin 2 \beta \sim 1 / \tan \beta$. For $\mu>0$ and fixed $m_{0}$, as $m_{1 / 2}$ increases both $\Delta_{B}$ and the RGE corrections to $B$ increase, yielding a relatively constant value for $\tan \beta$ when the growth of the term $-\Delta_{B}$ almost compensates the positive RGE corrections. For large values of $m_{1 / 2}$, the RGE corrections take over, resulting in the bending of the $\tan \beta$ contours. On the other hand, for $\mu<0$, the flipping of the sign of $\Delta_{B}$ described in the paragraph above results in different behaviour. In this case, as $m_{1 / 2}$ increases with fixed $m_{0}, \tan \beta$ always decreases.

In panel (a) of Fig. 2, the magnitude of the tree level value of $B$ at the weak scale increases with $m_{0}$, decreasing the value of $\tan \beta$. However, the loop correction is also growing, tending to increase $\tan \beta$. We see from the figure that $\tan \beta$ is first decreasing and then increasing as $m_{0}$ is increased. This behaviour is different from panel (b) of Fig. 2, where the tree level value of $B$ at the weak scale is decreasing with $m_{0}$, and dominates the determination of $\tan \beta$, which is now increasing monotonically.

At high values of $\hat{A}$ (and high $\tan \beta$ ), the off-diagonal elements in the squark mass matrix become large at large $m_{0}$. Therefore, we find no solutions which are phenomenologically viable above a certain value of $\hat{A}$. This is because the regions where the LSP is the $\widetilde{\tau}$ or the $\tilde{t}$ close off the parameter space ${ }^{5}$. In fact, this feature is generic in the CMSSM as shown in Fig. 3 of [3]. This effect is more severe at large $\tan \beta$, which further compounds the difficulty in going to large values of $\hat{A}$ in the type of models discussed here.

Finally, we note the absences of both the funnel and the focus-point regions. In the case of the funnel, this is due to the relatively small values of $\tan \beta$ allowed in the class of models considered here: we recall that the funnel region appears only for large $\tan \beta \gtrsim 45$ for $\mu>0$ and $\tan \beta \gtrsim 30$ for $\mu<0$ in the CMSSM.

To understand the absence of the focus-point region, we refer to [11], where it was shown that the position of the focus point is sensitive to the value of $A_{0}$. As $A_{0}$ is increased, the

\footnotetext{
${ }^{5}$ The neutralino-stop coannihilation region which occurs when $A_{0}$ is large in the small $\left(m_{1 / 2}, m_{0}\right)$ region [31] does not appear in our analysis because $A_{0}$ is still too small.
} 

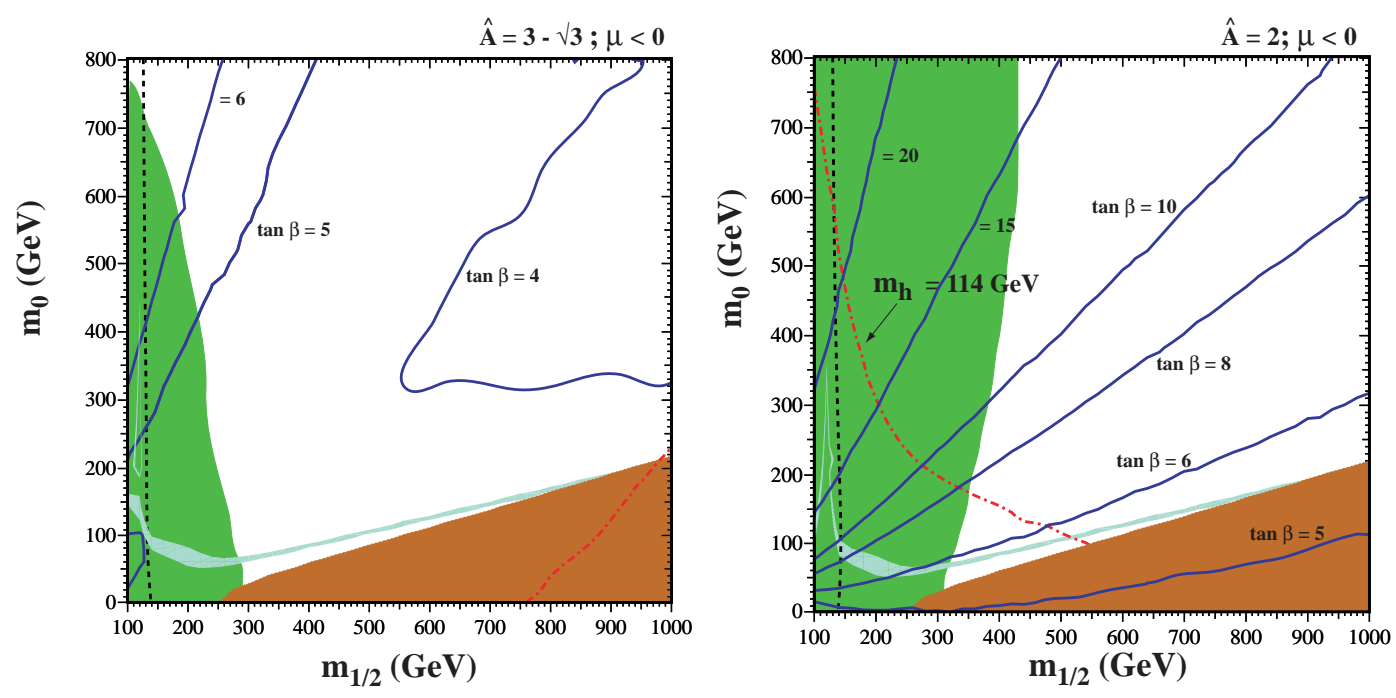

Figure 2: As in Fig. 1, but now for $\mu<0$ and the choices (a) $\hat{A}=3-\sqrt{3}, \hat{B}=\hat{A}-1$ and (b) $\hat{A}=2, \hat{B}=\hat{A}-1$ and $\mu<0$.

focus point is pushed up to higher values of $m_{0}$. Here, with $A_{0} \propto m_{0}$, the focus-point region recedes faster than $m_{0}$ if $\hat{A}$ is large enough, and is therefore never encountered. For small $\hat{A}$, $\tan \beta$ is small at large $m_{0}$, as shown in panel (b) of Fig. 1, so we do not find a focus point in this case, either. In addition, as can be inferred from the small disconnected segment of the $\tan \beta=10$ contour in the top left corner of panel (c), all the $\tan \beta$ contours loop back down to lower $m_{0}$ before reaching the focus-point region.

The above analysis shows that the 'bulk' $\Omega_{C D M} h^{2}$ region is almost completely excluded by the Higgs constraint, but a larger fraction would be allowed if we allowed a 2-GeV error in the CMSSM Higgs mass calculation, or if $m_{t}$ turns out to be significantly greater than $175 \mathrm{GeV}$. Almost all the coannihilation 'tail' region is allowed. As remarked on above, there is no 'funnel' region at large $m_{1 / 2}$ and $m_{0}$, nor any 'focus-point' region at large $m_{0}$.

\section{Bounds on $\tan \beta$}

It is clear from the previous figures that only limited ranges of $\tan \beta$ are consistent with the phenomenological constraints within any given pattern of supersymmetry breaking. We display in Fig. 3 the ranges of $\tan \beta$ allowed as a function of $\hat{A}$. For $\hat{B}=\hat{A}-1$ and $\mu>0$, as shown by the solid lines, we see that the upper and lower limits on $\tan \beta$ both increase monotonically with $\hat{A}$. We find consistent solutions to all the phenomenological constraints 
only for

$$
-1.9<\hat{A}<2.5
$$

over which range

$$
3.7<\tan \beta \lesssim 46
$$

Generally speaking, the range of $\tan \beta$ for any fixed value of $\hat{A}<0$ is very restricted, with larger ranges of $\tan \beta$ becoming allowed for $\hat{A}>0$. In the specific case of the simplest Polonyi model with positive $\hat{A}=3-\sqrt{3}$, we find

$$
11<\tan \beta<20
$$

whereas the range in $\tan \beta$ for the negative Polonyi model with $\hat{A}=\sqrt{3}-3$, is $4.4-4.6$. Furthermore, the difference between the upper and lower limits on $\tan \beta$ never exceeds $\sim 14$ for any fixed value of $\hat{A}$.

The corresponding results for $\mu<0$ are

$$
1.2<\hat{A}<4.8
$$

over which range

$$
4<\tan \beta \lesssim 26
$$

The range of $\hat{A}$ is shifted, and the range of $\tan \beta$ reduced, as compared to the case of $\mu>0$. In particular, the negative Polonyi model is disallowed and the positive version is allowed only for $\tan \beta \sim 4.15$.

\section{$7 \quad$ No-Scale Models}

We display in Fig. 4 the results of a similar analysis for the no-scale case $\hat{A}=\hat{B}=0$. For $\mu>0$, the allowed range of $\tan \beta$ is

$$
16<\tan \beta<30
$$

where the lower limit is provided by the Higgs search, and the upper limit is at the tip of the coannihilation 'tail'. For $\mu<0$, the same constraints allow just a small range around $\tan \beta \sim 4.8$. These two ranges are both shown as 'error bars' in Fig. 3.

However, the other no-scale condition $m_{0}=0$ is not allowed for either sign of $\mu$, the minimum being $m_{0} \simeq 62 \mathrm{GeV}$ for $\mu>0$ and $\tan \beta \simeq 16$. The fact that $m_{0} \neq 0$ is no surprise, since the same conclusion was reached previously without imposing the supplementary noscale conditions $\hat{A}=\hat{B}=0$ [13]. However, as we have already pointed out, the no-scale 


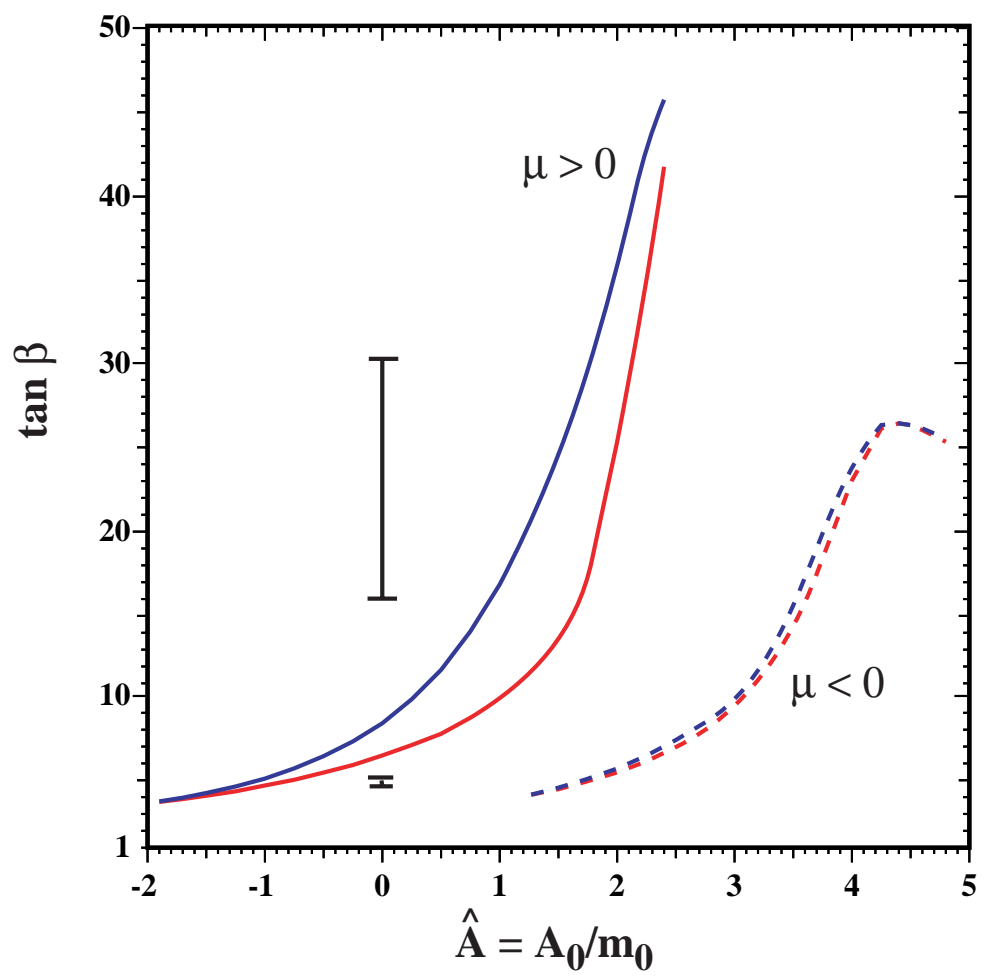

Figure 3: The ranges of $\tan \beta$ allowed if $\hat{B}=\hat{A}-1$ for $\mu>0$ (solid lines) and $\mu<0$ (dashed lines). The Polonyi model corresponds to $\hat{A} \simeq \pm 1.3$. Also shown as 'error bars' are the ranges of $\tan \beta$ allowed in the no-scale case $\hat{A}=\hat{B}=0$ for $\mu>0$ (upper) and $\mu<0$ (lower). 

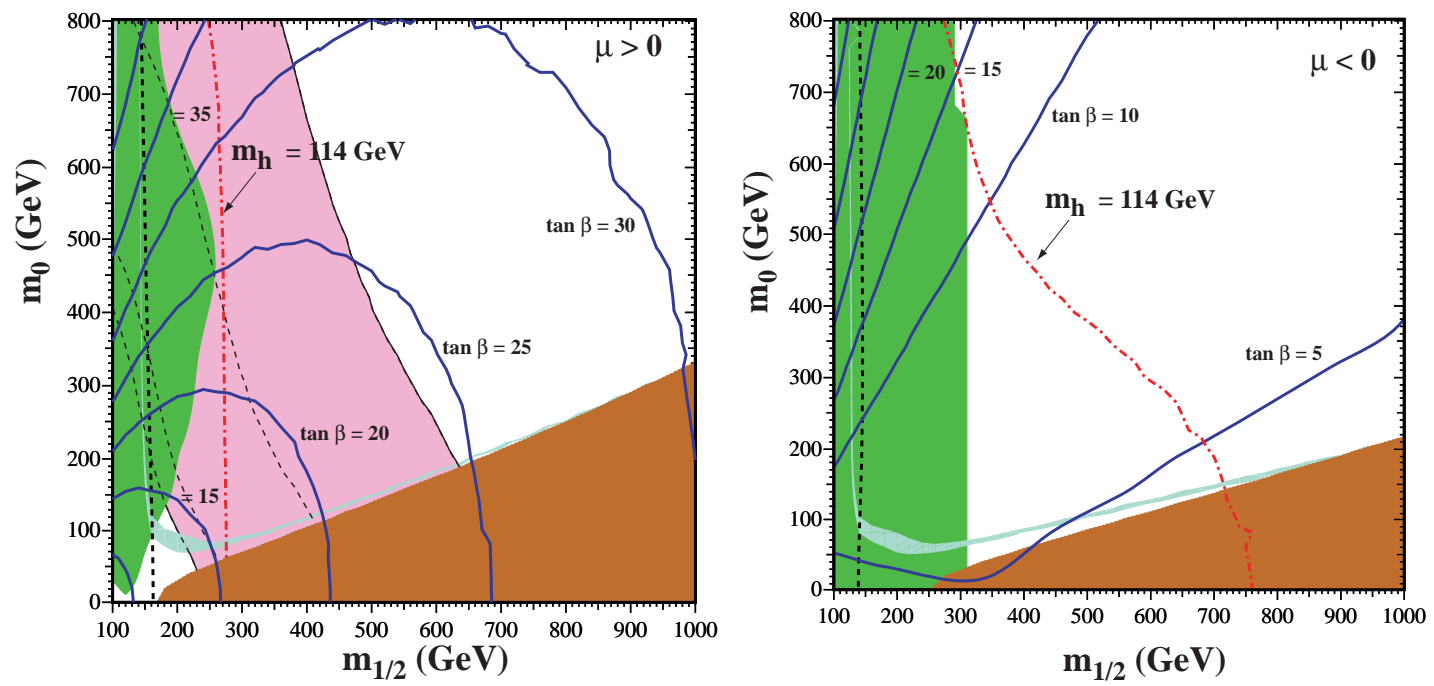

Figure 4: As in Fig. 1, for the no-scale cases $\hat{A}=0, \hat{B}=0$ and (a) $\mu>0$, (b) $\mu<0$.

boundary conditions should be interpreted as applying at the supergravity scale, so it is possible that $m_{0}, \hat{A}, \hat{B}$ all $\neq 0$, albeit small, at the GUT scale. We note that in this case, there is in fact a focus-point region at roughly the same position as in the CMSSM with $A_{0}=0$.

\section{Conclusions}

We have shown in this paper that only a restricted range of $\tan \beta$ is allowed in any specific pattern of supersymmetry breaking. We have illustrated this point by discussions of minimal supergravity models with $\hat{A}=\hat{B}+1$ and no-scale models with $\hat{A}=\hat{B}=0$, but the same comment would apply to other models of supersymmetry breaking not discussed here. Within the class of minimal supergravity models, we have selected in particular the simplest Polonyi model with $|\hat{A}|=3-\sqrt{3}$, but also discussed models with other values of $\hat{A}$, finding a rather restricted range, in particular for $\mu<0$.

One inference from our analysis is that an experimental determination of $\tan \beta$ could be a useful discriminator between different models of supersymmetry breaking. To understand the potential scope of this analysis tool, it would be necessary to study a wider class of models of supersymmetry breaking than those discussed here. 


\section{Acknowledgments}

The work of K.A.O., Y.S., and V.C.S. was supported in part by DOE grant DE-FG0294ER-40823.

\section{References}

[1] For reviews, see: H. P. Nilles, Phys. Rep. 110 (1984) 1; A. Brignole, L. E. Ibanez and C. Munoz, arXiv:hep-ph/9707209, published in Perspectives on supersymmetry, ed. G. L. Kane, pp. 125-148.

[2] J. Ellis, T. Falk, G. Ganis, K. A. Olive and M. Schmitt, Phys. Rev. D58 (1998) 095002 [arXiv:hep-ph/9801445].; J. R. Ellis, K. A. Olive and Y. Santoso, New J. Phys. 4 (2002) 32 [arXiv:hep-ph/0202110].

[3] J. R. Ellis, T. Falk, G. Ganis and K. A. Olive, Phys. Rev. D 62 (2000) 075010 [arXiv:hep$\mathrm{ph} / 0004169]$.

[4] J. R. Ellis, T. Falk, G. Ganis, K. A. Olive and M. Srednicki, Phys. Lett. B 510 (2001) 236 [arXiv:hep-ph/0102098].

[5] J. R. Ellis, K. A. Olive, Y. Santoso and V. C. Spanos, arXiv:hep-ph/0303043.

[6] A. B. Lahanas, D. V. Nanopoulos and V. C. Spanos, Phys. Rev. D 62 (2000) 023515 [arXiv:hep-ph/9909497]; V. Barger and C. Kao, Phys. Lett. B518 (2001) 117 [arXiv:hepph/0106189]; L. Roszkowski, R. Ruiz de Austri and T. Nihei, JHEP 0108 (2001) 024 [arXiv:hep-ph/0106334]; A. Djouadi, M. Drees and J. L. Kneur, JHEP 0108 (2001) 055 [arXiv:hep-ph/0107316]; R. Arnowitt and B. Dutta, arXiv:hep-ph/0211417; H. Baer, C. Balazs and A. Belyaev, JHEP 0203 (2002) 042 [arXiv:hep-ph/0202076]; T. Kamon, R. Arnowitt, B. Dutta and V. Khotilovich, arXiv:hep-ph/0302249; H. Baer, C. Balazs, A. Belyaev, T. Krupovnickas and X. Tata, arXiv:hep-ph/0304303.

[7] J. R. Ellis, T. Falk and K. A. Olive, Phys. Lett. B 444 (1998) 367 [arXiv:hepph/9810360]; J. R. Ellis, T. Falk, K. A. Olive and M. Srednicki, Astropart. Phys. 13 (2000) 181 [Erratum-ibid. 15 (2001) 413] [arXiv:hep-ph/9905481]; R. Arnowitt, B. Dutta and Y. Santoso, Nucl. Phys. B 606 (2001) 59 [arXiv:hep-ph/0102181].

[8] M. E. Gómez, G. Lazarides and C. Pallis, Phys. Rev. D D61 (2000) 123512 [arXiv:hepph/9907261]; Phys. Lett. B487 (2000) 313 [arXiv:hep-ph/0004028]; Nucl. Phys. B B638 
(2002) 165 [arXiv:hep-ph/0203131]; T. Nihei, L. Roszkowski and R. Ruiz de Austri, JHEP 0207 (2002) 024 [arXiv:hep-ph/0206266].

[9] M. Drees and M. M. Nojiri, Phys. Rev. D 47 (1993) 376 [arXiv:hep-ph/9207234]; H. Baer and M. Brhlik, Phys. Rev. D 53 (1996) 597 [arXiv:hep-ph/9508321]; H. Baer, M. Brhlik, M. A. Diaz, J. Ferrandis, P. Mercadante, P. Quintana and X. Tata, Phys. Rev. D 63 (2001) 015007 [arXiv:hep-ph/0005027]; A. B. Lahanas and V. C. Spanos, Eur. Phys. J. C 23 (2002) 185 [arXiv:hep-ph/0106345].

[10] J. L. Feng, K. T. Matchev and T. Moroi, Phys. Rev. Lett. 84 (2000) 2322; J. L. Feng, K. T. Matchev and T. Moroi, Phys. Rev. D61 (2000) 075005; J. L. Feng, K. T. Matchev and F. Wilczek, Phys. Lett. B482 (2000) 388.

[11] K. L. Chan, U. Chattopadhyay and P. Nath, Phys. Rev. D 58 (1998) 096004 [arXiv:hep$\mathrm{ph} / 9710473]$.

[12] E. Cremmer, S. Ferrara, C. Kounnas and D. V. Nanopoulos, Phys. Lett. B 133 (1983) 61; J. R. Ellis, A. B. Lahanas, D. V. Nanopoulos and K. Tamvakis, Phys. Lett. B 134 (1984) 429; A. B. Lahanas and D. V. Nanopoulos, Phys. Rept. 145 (1987) 1.

[13] J. R. Ellis, D. V. Nanopoulos and K. A. Olive, Phys. Lett. B 525 (2002) 308 [arXiv:hep$\mathrm{ph} / 0109288]$.

[14] E. Cremmer, B. Julia, J. Scherk, S. Ferrara, L. Girardello and P. Van Nieuwenhuizen, Phys. Lett. 79B (1978) 231; and Nucl. Phys. B147 (1979) 105; E. Cremmer, S. Ferrara, L. Girardello and A. Van Proeyen, Phys. Lett. 116B (1982) 231; and Nucl. Phys. B212 (1983) 413; R. Arnowitt, A.H. Chamseddine and P. Nath, Phys. Rev. Lett. 49 (1982) 970; 50 (1983) 232 and Phys. Lett. 121B (1983) 33; J. Bagger and E. Witten, Phys. Lett. 115B (1982) 202 and 118B (1982) 103; J. Bagger, Nucl. Phys. B211 (1983) 302.

[15] H.-P. Nilles, M. Srednicki and D. Wyler, Phys. Lett. $120 B$ (1983) 345; L.J. Hall, J. Lykken and S. Weinberg, Phys. Rev. D27 (1983) 2359.

[16] J. Polonyi, Budapest preprint KFKI-1977-93 (1977).

[17] R. Barbieri, S. Ferrara and C.A. Savoy, Phys. Lett. 119B (1982) 343.

[18] M. Endo, M. Matsumura and M. Yamaguchi, Phys. Lett. B 544 (2002) 161 [arXiv:hep$\mathrm{ph} / 0204349]$. 
[19] R. Arnowitt and P. Nath, Phys. Rev. D 46 (1992) 3981; V. D. Barger, M. S. Berger and P. Ohmann, Phys. Rev. D 49 (1994) 4908 [arXiv:hep-ph/9311269].

[20] W. de Boer, R. Ehret and D. I. Kazakov, Z. Phys. C 67 (1995) 647 [arXiv:hepph/9405342]; D. M. Pierce, J. A. Bagger, K. T. Matchev and R. J. Zhang, Nucl. Phys. B 491 (1997) 3 [arXiv:hep-ph/9606211].

[21] M. Carena, J. R. Ellis, A. Pilaftsis and C. E. Wagner, Nucl. Phys. B 625 (2002) 345 [arXiv:hep-ph/0111245].

[22] Joint LEP 2 Supersymmetry Working Group, Combined LEP Chargino Results, up to $208 \mathrm{GeV}$,

http://lepsusy.web.cern.ch/lepsusy/www/inos_moriond01/charginos_pub.html.

[23] Joint LEP 2 Supersymmetry Working Group, Combined LEP Selectron/Smuon/Stau Results, 183-208 GeV,

http://lepsusy.web.cern.ch/lepsusy/www/sleptons_summer02/slep_2002.html.

[24] LEP Higgs Working Group for Higgs boson searches, OPAL Collaboration, ALEPH Collaboration, DELPHI Collaboration and L3 Collaboration, Search for the Standard Model Higgs Boson at LEP, CERN-EP/2003-011, available from http://lephiggs . web.cern. ch/LEPHIGGS/papers/index.html.

[25] S. Heinemeyer, W. Hollik and G. Weiglein, Comput. Phys. Commun. 124 (2000) 76 [arXiv:hep-ph/9812320]; S. Heinemeyer, W. Hollik and G. Weiglein, Eur. Phys. J. C 9 (1999) 343 [arXiv:hep-ph/9812472].

[26] M.S. Alam et al., [CLEO Collaboration], Phys. Rev. Lett. 74 (1995) 2885 as updated in S. Ahmed et al., CLEO CONF 99-10; BELLE Collaboration, BELLE-CONF-0003, contribution to the 30th International conference on High-Energy Physics, Osaka, 2000. See also K. Abe et al., [Belle Collaboration], [arXiv:hep-ex/0107065]; L. Lista [BaBar Collaboration], [arXiv:hep-ex/0110010]; C. Degrassi, P. Gambino and G. F. Giudice, JHEP 0012 (2000) 009 [arXiv:hep-ph/0009337]; M. Carena, D. Garcia, U. Nierste and C. E. Wagner, Phys. Lett. B 499 (2001) 141 [arXiv:hep-ph/0010003]; P. Gambino and M. Misiak, Nucl. Phys. B 611 (2001) 338; D. A. Demir and K. A. Olive, Phys. Rev. D 65 (2002) 034007 [arXiv:hep-ph/0107329]; T. Hurth, arXiv:hep-ph/0212304.

[27] G. W. Bennett et al. [Muon g-2 Collaboration], Phys. Rev. Lett. 89 (2002) 101804 [Erratum-ibid. 89 (2002) 129903] [arXiv:hep-ex/0208001]. 
[28] M. Davier, S. Eidelman, A. Hocker and Z. Zhang, arXiv:hep-ph/0208177; see also K. Hagiwara, A. D. Martin, D. Nomura and T. Teubner, arXiv:hep-ph/0209187; F. Jegerlehner, unpublished, as reported in M. Krawczyk, arXiv:hep-ph/0208076.

[29] C. L. Bennett et al., arXiv:astro-ph/0302207; D. N. Spergel et al., arXiv:astroph/0302209.

[30] U. Chattopadhyay, A. Corsetti and P. Nath, arXiv:hep-ph/0303201; H. Baer and C. Balazs, arXiv:hep-ph/0303114; A. B. Lahanas and D. V. Nanopoulos, arXiv:hepph/0303130.

[31] J. R. Ellis, K. A. Olive and Y. Santoso, Astropart. Phys. 18 (2003) 395 [arXiv:hepph/0112113]; C. Boehm, A. Djouadi and M. Drees, Phys. Rev. D 62 (2000) 035012 [arXiv:hep-ph/9911496].

[32] J. Tabei and H. Hotta, arXiv:hep-ph/0208039. 